\title{
Variational Approaches for the Existence of Multiple Periodic Solutions of Differential Delay Equations
}

\author{
Rong Cheng' and Jianhua $\mathrm{Hu}^{2}$ \\ ${ }^{1}$ College of Mathematics and Physics, Nanjing University of Information Science and Technology, \\ Nanjing 210044, China \\ ${ }^{2}$ College of Science, University of Shanghai for Science and Technology, Shanghai 200093, China
}

Correspondence should be addressed to Rong Cheng, mathchr@163.com

Received 30 March 2009; Revised 15 December 2009; Accepted 11 January 2010

Academic Editor: John Mallet-Paret

Copyright (C) 2010 R. Cheng and J. Hu. This is an open access article distributed under the Creative Commons Attribution License, which permits unrestricted use, distribution, and reproduction in any medium, provided the original work is properly cited.

The existence of multiple periodic solutions of the following differential delay equation $x^{\prime}(t)=$ $-f(x(t-r))$ is established by applying variational approaches directly, where $x \in \mathbb{R}, f \in C(\mathbb{R}, \mathbb{R})$ and $r>0$ is a given constant. This means that we do not need to use Kaplan and Yorke's reduction technique to reduce the existence problem of the above equation to an existence problem for a related coupled system. Such a reduction method introduced first by Kaplan and Yorke in (1974) is often employed in previous papers to study the existence of periodic solutions for the above equation and its similar ones by variational approaches.

\section{Introduction}

We are concerned in this paper with the search for $4 r$-periodic solutions $(r>0)$ of a class of differential delay equations with the following form

$$
x^{\prime}(t)=-f(x(t-r))
$$

where $x \in \mathbb{R}, f \in C(\mathbb{R}, \mathbb{R})$, and $r>0$ is a given constant. Equation (1.1) occurs in variety of applications and describes many interesting types of phenomena. Taking $f(x)=-\delta+\sin x$, then (1.1) with the form

$$
x^{\prime}(t)=\delta-\sin (x(t-r)), \quad t \geq 0, \delta \geq 0, r \geq 0
$$


arises in the study of phase-locked loops which are widely used in communication systems [1]. Furthermore, many equations occurring in other fields of applications can be changed to the form of (1.1) by changing variables. For example, letting $x(t)=e^{u(t)}-1$, then the equation

$$
x^{\prime}(t)=\alpha x(t-1)(1+x(t))
$$

can be changed to the form of (1.1). Equation (1.3) was first proposed by Cunningham [2] as a nonlinear population growth model. Later Wright in [3] mentioned it as arising in the application of probability methods to the theory of asymptotic prime number density. For applications of (1.3) and its similar ones on ecology, one may see [4].

Because of extensive applications, (1.1) and (1.3) have been studied by many authors through various methods [1-3, 5-23]. In 1962, Jones in his paper of [13] considered (1.3) and obtained the existence of periodic solutions of (1.3) by applying fixed point theory. Nussbaum [14] also used fixed point theory [24] for the truncated cones of Krasnosel'skii to give an existence result on periodic solutions.

Besides various fixed point theorems, the global Hopf bifurcation theorem for differential delay equations introduced by Chow and Mallet-Paret [25], qualitative theory of ordinary differential equations, some well-known results on the existence of closed orbits for Hamiltonian vector fields, coincidence degree theory introduced by Mawhin [26, 27], and the Poincaré-Bendixson theorem are proved to be very useful tools in searching for periodic solutions of differential delay (1.1) and (1.3).

A different approach for establishing the existence of periodic solutions for the differential delay (1.1) was introduced by Kaplan and Yorke in their paper in [7]. Employing the method, they could reduce the search for periodic solutions of (1.1) to the problem of seeking periodic solutions for a related system of ordinary differential equations, which is called the coupled system to (1.1). Following the reduction idea of Kaplan and Yorke, Li and He $[17,18]$ were able to translate (1.1) with more than one delay to a coupled Hamiltonian system. Then they used variational approaches to study the coupled Hamiltonian system and obtained some existence results of multiple periodic solutions of the equations. This proves that variational approaches $[28,29]$ also are very powerful tools to study periodic solutions of (1.1), (1.3), and their similar ones.

Recently, Guo and Yu [19] do not use Kaplan and Yorke's reduction technique and apply variational methods directly to study the existence of multiple periodic solutions of (1.1) with $x$ and $f$ being vectors in $\mathbb{R}^{n}$. That is to say they do not reduce the existence problem of (1.1) to an existence problem of a related coupled Hamiltonian system. By applying the pseudo-index introduced by Benci in [30], they obtained a sufficient condition on the existence and multiplicity of periodic solutions for (1.1). To the author's knowledge, this is the first time in which the existence of periodic solutions of (1.1) is studied by variational methods directly.

Let us say some words about the two methods. The advantage of direct variational method is that the function $f$ could be a vector in $\mathbb{R}^{n}$ while $f$ is only being a scalar in Kaplan and Yorke's reduction method. But Kaplan and Yorke's reduction method can deal with (1.1) with more than two delays while direct variational method used by Guo and Yu only admits one delay in (1.1).

Motivated by the work of Guo and Yu, in this paper we will also use variational approaches directly to study the existence of periodic solutions of (1.1). But our arguments are quite different from theirs. Throughout this paper, we make the following assumptions. 
(H1) $f(x) \in C(\mathbb{R}, \mathbb{R})$, and for any $x \in \mathbb{R}, f(x)+f(-x)=0$, that is, $f(x)$ is odd.

(H2) $f(x)$ satisfies

$$
\lim _{x \rightarrow 0} \frac{f(x)}{x}=-\alpha_{0} \in \mathbb{R}, \quad \lim _{x \rightarrow \infty} \frac{f(x)}{x}=-\alpha_{\infty} \in \mathbb{R} .
$$

In order to state our main result, we need the following definition.

Definition 1.1. For each $\alpha \in \mathbb{R}$, define

$$
\begin{aligned}
& \mu(\alpha)=\sum_{m=1}^{\infty}\left\{\sigma^{-}\left[(-1)^{m} \frac{\pi}{2 r}(2 m-1)-\alpha\right]-\sigma^{-}\left((-1)^{m}\right)\right\} \\
& v(\alpha)=\sum_{m=1}^{\infty} \sigma^{0}\left[(-1)^{m} \frac{\pi}{2 r}(2 m-1)-\alpha\right]
\end{aligned}
$$

where the two functions $\sigma^{-}(t)$ and $\sigma^{0}(t)$ are given by

$$
\sigma^{-}(t)=\left\{\begin{array}{ll}
1, & t<0, \\
0, & t \geq 0,
\end{array} \quad \sigma^{0}(t)= \begin{cases}1, & t=0 \\
0, & t \neq 0\end{cases}\right.
$$

It is easy to see that $\mu(\alpha)$ and $v(\alpha)$ are well defined. Let $\mu_{0}=\mu\left(\alpha_{0}\right), \mu_{\infty}=\mu\left(\alpha_{\infty}\right)$, $v_{0}=v\left(\alpha_{0}\right)$, and $v_{\infty}=v\left(\alpha_{\infty}\right)$. Then our main result states as follows.

Theorem 1.2. Suppose that $f$ satisfies (H1) and (H2). Then the following conclusions are true.

(i) If $\alpha_{\infty} \notin(\pi / 2 r)(2 \mathbb{Z}-1)$, then (1.1) possesses at least $\max \left\{\mu_{0}-\mu_{\infty}, \mu_{\infty}-\mu_{0}-v_{0}\right\}$ nontrivial geometrically different $4 r$-periodic solutions.

(ii) If $\alpha_{\infty} \in(\pi / 2 r)(2 \mathbb{Z}-1),\left|f(x)+\alpha_{\infty} x\right|$ is bounded, and $\int_{0}^{x} f(y) d y+(1 / 2) \alpha_{\infty} x^{2}$ converges to $-\infty$ as $|x| \rightarrow \infty$, then the conclusion of (i) also holds.

Remark 1.3. We say that two solutions of (1.1) are geometrically different if one cannot be obtained by time rescaling of the other. We will use [31, Theorem 2.4] to prove the main result.

\section{Variational Functional on Hilbert Space}

In this section, we will construct a variational functional of (1.1) defined on a suitable Hilbert space such that finding $4 r$-periodic solutions of (1.1) is equivalent to seeking critical points of the functional.

We work in the Hilbert space $H=H^{1 / 2,2}\left(S^{1}, \mathbb{R}\right)$ which consists of those functions $x \in L^{2}\left(S^{1}, \mathbb{R}\right)$ having weak derivative $x^{\prime} \in L^{2}\left(S^{1}, \mathbb{R}\right)$. The simplest way to introduce this space seems as follows. Each function $x \in L^{2}\left(S^{1}, \mathbb{R}\right)$ has the following Fourier expansion:

$$
x(t)=a_{0}+\sum_{m=1}^{+\infty}\left(a_{m} \cos \frac{\pi}{2 r} m t+b_{m} \sin \frac{\pi}{2 r} m t\right)
$$


where $a_{m}, b_{m} \in \mathbb{R}$. $H$ is the set of those functions satisfying

$$
\|x\|^{2}=4 r a_{0}^{2}+2 r \sum_{m=1}^{+\infty} m\left(a_{m}^{2}+b_{m}^{2}\right)<+\infty
$$

With this norm $\|\cdot\|, H$ is a Hilbert space with the following inner product:

$$
\langle x, y\rangle=4 r a_{0} a_{0}^{\prime}+2 r \sum_{m=1}^{\infty} m\left(a_{m} a_{m}^{\prime}+b_{m} b_{m}^{\prime}\right)
$$

where $y=a_{0}^{\prime}+\sum_{m=1}^{+\infty}\left(a_{m}^{\prime} \cos (\pi / 2 r) m t+b_{m}^{\prime} \sin (\pi / 2 r) m t\right)$.

For each $x \in H$, we define a functional $I: H \rightarrow \mathbb{R}$ by

$$
I(x)=\int_{0}^{4 r} \frac{1}{2} x^{\prime}(t+r) x(t) d t+\int_{0}^{4 r} F(x(t)) d t
$$

where $F(x)=\int_{0}^{x} f(y) d y$.

By Riesz representation theorem, $H$ identifies its dual space $H^{*}$. Then we define an operator $J: H \rightarrow H^{*}=H$ by extending the bilinear form

$$
\langle J x, y\rangle=\int_{0}^{4 r} x^{\prime}(t+r) y(t) d t, \quad \forall x, y \in H
$$

In fact, define a map $L: H \times H \rightarrow H$ by $L(x, y)=\langle J x, y\rangle$. Then $L$ is linear in $x$ and $y$, respectively, and there exists a positive number $M>0$ such that

$$
\|L(x, y)\| \leq M\|x\|\|y\| .
$$

Thus $L$ is a bilinear form from $H \times H \rightarrow H$. So $J$ is a bounded linear operator on $H$ and $\operatorname{ker} J=\mathbb{R}$.

For any $x \in H$, define a mapping $K: H \rightarrow \mathbb{R}$ as

$$
K(x)=\int_{0}^{4 r} F(x(t)) d t .
$$

Then the functional $I$ can be rewritten as

$$
I(x)=\frac{1}{2}\langle J x, x\rangle+K(x), \quad \forall x \in H .
$$

According to a standard argument in [30], one has, for any $x, y \in H$,

$$
\left\langle I^{\prime}(x), y\right\rangle=\int_{0}^{4 r} \frac{1}{2}\left(x^{\prime}(t+r)-x^{\prime}(t-r)\right) y(t) d t+\int_{0}^{4 r} f(x(t)) y(t) d t .
$$


Moreover $K^{\prime}: H \rightarrow H$ is a compact operator defined by

$$
\left\langle K^{\prime}(x), y\right\rangle=\int_{0}^{4 r} f(x(t)) y(t) d t
$$

Our aim is to reduce the existence of periodic solutions of (1.1) to the existence of critical points of $I$. For this we introduce a shift operator $\Gamma: H \rightarrow H$ defined by

$$
\Gamma x(t)=x(t+r) .
$$

It is easy to compute that $\Gamma$ is bounded and linear. Moreover $\Gamma$ is isometric, that is, $\|\Gamma x\|=\|x\|$ and $\Gamma^{4}=i d$, where $i d$ denotes the identity mapping on $H$.

Write

$$
E=\left\{x \in H: \Gamma^{2} x(t)=-x(t)\right\} .
$$

By a direct computation, solutions of the Euler equation of $I$ in $E$ are exactly solutions of (1.1).

Lemma 2.1. As a closed subspace of $H, E$ has the following property:

$$
E=\left\{x(t)=\sum_{m=1}^{+\infty}\left(a_{2 m-1} \cos \frac{\pi}{2 r}(2 m-1) t+b_{2 m-1} \sin \frac{\pi}{2 r}(2 m-1) t\right)\right\} .
$$

Proof. It is easy to see that $E$ is a closed subspace of $H$. For any $x(t)=a_{0}+$ $\sum_{m=1}^{+\infty}\left(a_{m} \cos (\pi / 2 r) m t+b_{m} \sin (\pi / 2 r) m t\right) \in E, \Gamma^{2} x=-x$ implies that $x(t+2 r)=-x(t)$. Then one has

$$
a_{0}=-a_{0}, \quad a_{m}=(-1)^{m+1} a_{m}, \quad b_{m}=(-1)^{m+1} b_{m},
$$

that is,

$$
a_{m}=b_{m}=0 \text { for even } m \text {. }
$$

Thus, for any $x(t) \in E$,

$$
x(t)=\sum_{m=1}^{+\infty}\left(a_{2 m-1} \cos \frac{\pi}{2 r}(2 m-1) t+b_{2 m-1} \sin \frac{\pi}{2 r}(2 m-1) t\right) .
$$

Hence we get the conclusion. 
If we restrict $J$ on $E$, a direct check shows that $J$ over $E$ is self-adjoint. For any $x \in E$, let $J x(t)=\sum_{m=1}^{+\infty}\left(a_{2 m-1}^{J} \cos (\pi / 2 r)(2 m-1) t+b_{2 m-1}^{J} \sin (\pi / 2 r)(2 m-1) t\right)$. Let $y=$ $\sum_{m=1}^{+\infty}\left(a_{2 m}^{\prime} \cos (\pi / 2 r)(2 m-1) t+b_{2 m-1}^{\prime} \sin (\pi / 2 r)(2 m-1) t\right)$. Then

$$
\begin{gathered}
x^{\prime}(t+r)=\sum_{m=1}^{+\infty}\left[(-1)^{m} \frac{\pi}{2 r}(2 m-1)\left(a_{2 m-1} \cos \frac{\pi}{2 r}(2 m-1) t+b_{2 m-1} \sin \frac{\pi}{2 r}(2 m-1) t\right)\right], \\
\int_{0}^{4 r} x^{\prime}(t+r) y(t) d t=(-1)^{m}\left[\pi(2 m-1) a_{2 m-1} a_{2 m-1}^{\prime}+\pi(2 m-1) b_{2 m-1} b_{2 m-1}^{\prime}\right] .
\end{gathered}
$$

According to the definition of the inner product in $E$, we have

$$
a_{2 m-1}^{J}=(-1)^{m} \frac{\pi}{2 r} a_{2 m-1}, \quad b_{2 m-1}^{J}=(-1)^{m} \frac{\pi}{2 r} b_{2 m-1},
$$

that is,

$$
J x(t)=\sum_{m=1}^{+\infty}\left[(-1)^{m} \frac{\pi}{2 r}\left(a_{2 m-1} \cos \frac{\pi}{2 r}(2 m-1) t+b_{2 m-1} \sin \frac{\pi}{2 r}(2 m-1) t\right)\right] .
$$

Let $\left.I\right|_{E}$ denote the restriction of $I$ on $E$. Then we have the following lemma.

Lemma 2.2. Critical points of $\left.I\right|_{E}$ over E are critical points of I on $H$.

Proof. Note that any $x \in E$ is 4 rperiodic and $f$ is odd. It is enough for us to prove that $\left\langle I^{\prime}(x), y\right\rangle=0$ for any $y \in H$ and $x$ being a critical point of $I$ in $E$.

For any $y \in H$, we have

$$
\begin{aligned}
\left\langle\Gamma^{2} I^{\prime}(x), y\right\rangle & =\left\langle\Gamma^{2} J x, y\right\rangle+\left\langle\Gamma^{2} K^{\prime}(x), y\right\rangle \\
& =\left\langle J x, \Gamma^{-2} y\right\rangle+\left\langle K^{\prime}(x), \Gamma^{-2} y\right\rangle \\
& =\int_{0}^{4 r} x^{\prime}(t+r) y(t-2 r) d t+\int_{0}^{4 r} f(x(t)) y(t-2 r) d t \\
& =-\int_{0}^{4 r} x^{\prime}(t+r) y(t) d t-\int_{0}^{4 r} f(x(t)) y(t) d t \\
& =\left\langle-I^{\prime}(x), y\right\rangle .
\end{aligned}
$$

This yields $\Gamma^{2} I^{\prime}(x)=-I^{\prime}(x)$, that is, $I^{\prime}(x) \in E$.

Suppose that $x(t)$ is a critical point of $I$ in $E$. We only need to show that $\left\langle I^{\prime}(x), y\right\rangle=0$ for any $y \in H$. Writing $y=y_{1} \oplus y_{2}$ with $y_{1} \in E, y_{2} \in E^{\perp}$ and noting that $I^{\prime}(x) \in E$, one has

$$
\left\langle I^{\prime}(x), y\right\rangle=\left\langle I^{\prime}(x), y_{1}\right\rangle+\left\langle I^{\prime}(x), y_{2}\right\rangle=0+0=0
$$

The proof is complete. 
Remark 2.3. By Lemma 2.2, we only need to consider $\left.I\right|_{E}$. Therefore in the following $I$ will be assumed on $E$.

For any $\alpha \in \mathbb{R}$, we define an operator $L_{\alpha}: H \rightarrow H$ by

$$
\left\langle L_{\alpha} x, y\right\rangle=\int_{0}^{4 r} \alpha x(t) y(t) d t
$$

It is not difficult to see that $L$ is a bounded self-adjoint linear mapping on $E$. Furthermore, for any $x(t) \in E$, let $L_{\alpha} x(t)=\sum_{m=1}^{+\infty}\left(a_{2 m-1}^{L_{\alpha}} \cos (\pi / 2 r)(2 m-1) t+\right.$ $\left.b_{2 m-1}^{L_{\alpha}} \sin (\pi / 2 r)(2 m-1) t\right)$. Let $y=\sum_{m=1}^{+\infty}\left(a_{2 m-1}^{\prime} \cos (\pi / 2 r)(2 m-1) t+b_{2 m-1}^{\prime} \sin (\pi / 2 r)(2 m-1) t\right)$. Then

$$
\int_{0}^{4 r} \alpha x(t) y(t) d t=2 r \alpha a_{2 m-1} a_{2 m-1}^{\prime}+2 r \alpha b_{2 m-1} b_{2 m-1}^{\prime}
$$

Similar to (2.19), we have

$$
a_{2 m-1}^{L_{\alpha}}=\frac{\alpha}{2 m-1} a_{2 m-1}, \quad b_{2 m-1}^{L_{\alpha}}=\frac{\alpha}{2 m-1} b_{2 m-1},
$$

that is,

$$
L_{\alpha} x(t)=\sum_{m=1}^{+\infty} \frac{\alpha}{2 m-1}\left[a_{2 m-1} \cos \frac{\pi}{2 r}(2 m-1) t+b_{2 m-1} \sin \frac{\pi}{2 r}(2 m-1) t\right]
$$

Set

$$
\begin{gathered}
E(m)=\left\{x \in E: x(t)=a_{2 m-1} \cos \frac{\pi}{2 r}(2 m-1) t+b_{2 m-1} \sin \frac{\pi}{2 r}(2 m-1) t\right\}, \\
E_{n}=E(1) \oplus E(2) \oplus \cdots \oplus E(n) .
\end{gathered}
$$

Let $P_{n}$ be the orthogonal projection from $E$ to $E_{n}$, then one has

$$
P_{n} x(t)=\sum_{m=1}^{n}\left(a_{2 m-1} \cos \frac{\pi}{2 r}(2 m-1) t+b_{2 m-1} \sin \frac{\pi}{2 r}(2 m-1) t\right) .
$$

Denote by $M^{+}\left(J-L_{\alpha}\right), M^{-}\left(J-L_{\alpha}\right)$, and $M^{0}\left(J-L_{\alpha}\right)$ the positive definite, the negative definite, and the null subspace of the self-adjoint operator $J-L_{\alpha}$, respectively. Then we have the following lemma. 
Lemma 2.4. For $m \geq 1$, let $\lambda_{m}=(-1)^{m}(\pi / 2 r)(2 m-1)-\alpha$. Then the following conclusions hold:

$$
\begin{aligned}
& M^{+}\left(J-L_{\alpha}\right)=\bigoplus_{m=1, \lambda_{m}>0}^{\infty} E(m), \\
& M^{-}\left(J-L_{\alpha}\right)=\bigoplus_{m=1, \lambda_{m}<0}^{\infty} E(m), \\
& M^{0}\left(J-L_{\alpha}\right)=\bigoplus_{m=1, \lambda_{m}=0}^{\infty} E(m) .
\end{aligned}
$$

Moreover for $m$ large enough,

$$
\begin{aligned}
& \operatorname{dim} M^{-}\left(P_{m}\left(J-L_{\alpha}\right) P_{m}\right)=2 \mu(\alpha)+2 \sum_{j=1}^{m} \sigma^{-}\left((-1)^{j}\right), \\
& \operatorname{dim} M^{0}\left(P_{m}\left(J-L_{\alpha}\right) P_{m}\right)=2 v(\alpha) .
\end{aligned}
$$

Proof. For $x_{m}=a_{2 m-1} \cos (\pi / 2 r)(2 m-1) t+b_{2 m-1} \sin (\pi / 2 r)(2 m-1) t$, consider the following eigenvalue problem:

$$
\left(J-L_{\alpha}\right) x_{m}=\lambda x_{m}
$$

From (2.19) and (2.25), one has

$$
\begin{aligned}
& {\left[(-1)^{m} \frac{\pi}{2 r}-\frac{\alpha}{2 m-1}\right] a_{2 m-1}=\lambda a_{2 m-1},} \\
& {\left[(-1)^{m} \frac{\pi}{2 r}-\frac{\alpha}{2 m-1}\right] b_{2 m-1}=\lambda b_{2 m-1} .}
\end{aligned}
$$

The above two equalities show that $J-L_{\alpha}$ is positive definite, negative definite, and null on $E(m)$ if and only if $\lambda_{m}=(-1)^{m}(\pi / 2 r)(2 m-1)-\alpha$ is positive, negative, and zero, respectively.

By Definition 1.1 and for $m$ large enough,

$$
\begin{aligned}
\operatorname{dim} M^{-}\left(P_{m}\left(J-L_{\alpha}\right) P_{m}\right) & =2 \sum_{j=1}^{m} \sigma^{-}\left[(-1)^{m} \frac{\pi}{2 r}(2 m-1)-\alpha\right] \\
& =2 \sum_{j=1}^{m} \sigma^{-}\left[(-1)^{m} \frac{\pi}{2 r}(2 m-1)-\alpha\right]-2 \sum_{j=1}^{m} \sigma^{-}\left((-1)^{j}\right)+2 \sum_{j=1}^{m} \sigma^{-}\left((-1)^{j}\right) \\
& =2 \mu(\alpha)+2 \sum_{j=1}^{m} \sigma^{-}\left((-1)^{j}\right) .
\end{aligned}
$$

The last equality of Lemma 2.4 is obvious. The proof is complete. 


\section{Proof of the Main Result}

In this section, we will use [31, Theorem 2.4] to prove our main result. To state the theorem, we need the following notation and definition. For $\theta \in S^{1}$ and $x \in E$, define an action $T$ on $E$ by

$$
T(\theta) x(t)=x(t+\theta) .
$$

A direct computation shows that

$$
\operatorname{Fix}\left(S^{1}\right)=\left\{x \in E: T(\theta)(x)=x, \forall \theta \in S^{1}\right\}=\{0\} .
$$

Definition 3.1. I satisfies $(P S)_{c}$ condition on $E$ if every sequence $\left\{x_{m}\right\} \subset E$ with $I\left(x_{m}\right) \rightarrow c$ and $I^{\prime}\left(x_{m}\right) \rightarrow 0$ possesses a convergent subsequence. as follows.

Write $L_{0}=L_{\alpha_{0}}$ and $L_{\infty}=L_{\alpha_{\infty}}$. Then [31, Theorem 2.4] applied to $I$ on $E$ can be stated

Lemma 3.2. Suppose that there exist two closed $S^{1}$-invariant linear subspace of $E, V$ and, $W$ and a positive number $s>0$ such that

(1) $(V+W)$ is closed and of finite codimension in $E$,

(2) $L(W) \subseteq W$ with $A=J-L_{0}$ or $A=J-L_{\infty}$,

(3) there exists $\beta_{0} \in \mathbb{R}$ such that

$$
\inf _{x \in V} I(x) \geq \beta_{0}
$$

(4) there exists $\beta_{\infty} \in \mathbb{R}$ such that

$$
I(x) \leq \beta_{\infty}<I(0), \quad \forall x \in W \cap B_{s}=\{x \in W:\|x\|=s\},
$$

(5) I satisfies (PS) $)_{c}$ condition for $\beta_{0} \leq c \leq \beta_{\infty}$.

Then I possesses at least $(1 / 2)[\operatorname{dim}(V \cap W)-\operatorname{codim}(V+W)]$ geometrically distinct critical orbits in $I^{-1}\left(\left[\beta_{0}, \beta_{\infty}\right]\right)$.

Lemma 3.3. Assume that (H1), (H2), and the assumptions of (i) or (ii) of Theorem 1.2 hold. Then I satisfies $(P S)_{c}$ condition on $E$.

Proof. We first show that $\left\{x_{m}\right\} \subset E$ is bounded. Assume that $\left\{x_{m}\right\}$ is not bounded. By passing to a subsequence, if necessary, we may assume that $\left\|x_{m}\right\| \rightarrow \infty$ as $m \rightarrow \infty$.

Case (i). Suppose that $\alpha_{\infty} \notin(\pi / 2 r)(2 \mathbb{Z}-1)$. Then by Definition $1.1, v_{\infty}=0$, which yields $M^{0}\left(J-L_{\infty}\right)=0$. Thus $J-L_{\infty}$ has bounded inverse, that is, $\exists \kappa>0$ such that

$$
\left\|\left(J-L_{\infty}\right)^{-1}\right\| \leq \frac{1}{\kappa}
$$


For any $x \in E$, define

$$
I_{0}(x)=K(x)+\frac{1}{2}\left\langle L_{0} x, x\right\rangle, \quad I_{\infty}(x)=K(x)+\frac{1}{2}\left\langle L_{\infty} x, x\right\rangle .
$$

Then $I(x)$ can be written as

$$
I(x)=\frac{1}{2}\left\langle\left(J-L_{0}\right) x, x\right\rangle+I_{0}(x), \quad \text { or } \quad I(x)=\frac{1}{2}\left\langle\left(J-L_{\infty}\right) x, x\right\rangle+I_{\infty}(x) .
$$

By condition (H2), we have

$$
\frac{\left\|I_{\infty}^{\prime}(x)\right\|}{\|x\|} \longrightarrow 0, \quad \text { as }\|x\| \longrightarrow \infty
$$

This means that for any $0<\gamma<\kappa$ there exists $\delta>0$ such that

$$
\left\|I_{\infty}^{\prime}(x)\right\| \leq \gamma\|x\|, \quad \text { as }\|x\| \geq \delta .
$$

Note that $\left(J-L_{\infty}\right) x_{m}-I^{\prime}\left(x_{m}\right)=-I_{\infty}^{\prime}\left(x_{m}\right)$. We have

$$
\begin{aligned}
\left\|x_{m}\right\|-\left\|\left(J-L_{\infty}\right)^{-1} I^{\prime}\left(x_{m}\right)\right\| & \leq\left\|x_{m}-\left(J-L_{\infty}\right)^{-1} I^{\prime}\left(x_{m}\right)\right\| \\
& =\left\|\left(J-L_{\infty}\right)^{-1}\left(\left(J-L_{\infty}\right) x_{m}-I^{\prime}\left(x_{m}\right)\right)\right\| \\
& \leq\left\|\left(J-L_{\infty}\right)^{-1}\right\|\left\|I_{\infty}^{\prime}\left(x_{m}\right)\right\| \\
& \leq \frac{1}{\mathcal{K}} \gamma\left\|x_{m}\right\| .
\end{aligned}
$$

Then by the above estimates,

$$
\left\|x_{m}\right\| \leq \frac{\kappa}{\kappa-\gamma}\left\|\left(J-L_{\infty}\right)^{-1}\right\|\left\|I^{\prime}\left(x_{m}\right)\right\| \leq \frac{\kappa}{\kappa-\gamma} \frac{1}{\mathcal{\kappa}}\left\|I^{\prime}\left(x_{m}\right)\right\| \longrightarrow 0, \quad \text { as } m \longrightarrow \infty .
$$

This contradicts $\left\|x_{m}\right\| \rightarrow \infty$ as $m \rightarrow \infty$. Hence $\left\|x_{m}\right\|$ is bounded.

Now we show that $\left\|x_{m}\right\|$ has a convergent subsequence. Notice that $P_{0}: E \rightarrow \operatorname{ker} J=\mathbb{R}$ is of finite rank and $K^{\prime}: H \rightarrow H$ is compact. Therefore we may suppose that

$$
K^{\prime}\left(x_{m}\right) \longrightarrow y, \quad P_{0} x_{m} \longrightarrow z \quad \text { in } E \text { as } m \longrightarrow \infty
$$

Since $J+P_{0}$ has continuous inverse $\left(J+P_{0}\right)^{-1}$, it follows from

$$
\left(J+P_{0}\right) x_{m}=I^{\prime}\left(x_{m}\right)-K^{\prime}\left(x_{m}\right)+P_{0} x_{m}
$$


that

$$
x_{m}=\left(J+P_{0}\right)^{-1}\left(I^{\prime}\left(x_{m}\right)-K^{\prime}\left(x_{m}\right)+P_{0} x_{m}\right) \longrightarrow\left(J+P_{0}\right)^{-1}(z-y) \quad \text { as } m \longrightarrow \infty .
$$

Thus $\left\{x_{m}\right\}$ has a convergent subsequence.

Case (ii). Assume that $\left\{x_{m}\right\}$ is not bounded. Since $\left|f(x)+\alpha_{\infty} x\right|$ is bounded, there exists a constant $C>0$ such that

$$
\left\|I_{\infty}^{\prime}(x)\right\|<C, \quad \forall x \in E
$$

Observe that $\left(J-L_{\infty}\right) x=I^{\prime}(x)-I_{\infty}^{\prime}(x)$ and $I^{\prime}\left(x_{m}\right) \rightarrow 0$ as $m \rightarrow \infty$,

$$
\left\|\left(J-L_{\infty}\right) x_{m}\right\| \leq\left\|I^{\prime}\left(x_{m}\right)\right\|+\left\|I_{\infty}^{\prime}\left(x_{m}\right)\right\| \leq C+1 \quad \text { as } m \longrightarrow \infty
$$

Since $\left\|x_{m}\right\| \rightarrow \infty$ and $F\left(x_{m}\right)+(1 / 2) \alpha_{\infty} x_{m}^{2}$ converges to $-\infty$ as $m \rightarrow \infty$,

$$
I_{\infty}\left(x_{m}\right)=I\left(x_{m}\right)-\frac{1}{2}\left\langle\left(J-L_{\infty}\right) x_{m}, x_{m}\right\rangle>I\left(x_{m}\right)-\frac{C+1}{2}\left\|x_{m}\right\| .
$$

Let $m \rightarrow \infty$, then (3.17) implies $-\infty>-\infty$. This is a contradiction. Therefore $\left\{x_{m}\right\}$ is bounded. With the same discussion as that of Case (i), we can prove that $\left\{x_{m}\right\}$ has a convergent subsequence. The proof is complete.

Now we are ready to prove our main result.

Proof of Theorem 1.2. Since $x(t) \in E$ is periodic and $F(x(t))$ is independent of $t, I$ is $S^{1}$ invariant and $I^{\prime}$ is $S^{1}$-equivariant according to the action $T$ on $S^{1}$.

Case (i). Assume first $\mu_{0}-\mu_{\infty}>0$. Take two subspaces $V$ and $W$ as

$$
V=M^{+}\left(J-L_{\infty}\right) \oplus M^{0}\left(J-L_{\infty}\right), \quad W=M^{-}\left(J-L_{0}\right) .
$$

Since inner product is continuous and $\operatorname{codim}(V+W)=0$, the assumption (1) of Lemma 3.2 holds. Let $A=J-L_{0}$. We can check easily that $A(W) \subseteq W$. By Lemma 3.3, I satisfies $(P S)_{c}$ condition. Thus the assumptions (2) and (5) of Lemma 3.2 hold.

By the proof of Lemma 3.3, $M^{0}\left(J-L_{\infty}\right)=0$. For $x \in V=M^{+}\left(J-L_{\infty}\right)$, there is a suitable constant $c_{1}>0$ such that

$$
\left\langle\left(J-L_{\infty}\right) x, x\right\rangle \geq c_{1}\|x\|^{2}
$$

Then by (3.8), for any $x \in V$,

$$
I(x)=\frac{1}{2}\left\langle\left(J-L_{\infty}\right) x, x\right\rangle+I_{\infty}(x) \geq \frac{1}{2} c_{1}\|x\|^{2}-c_{2}\|x\|,
$$

where $I_{\infty}(x)$ is defined in (3.6). Hence there exists $\beta_{0}<0$ such that the assumption (3) of Lemma 3.2 holds. 
By $(H 2)$, we have

$$
I_{0}(0)=0, \quad \frac{\left\|I_{0}^{\prime}(x)\right\|}{\|x\|} \longrightarrow 0 \quad \text { as }\|x\| \longrightarrow 0,
$$

where $I_{0}(x)$ is defined in (3.6). For any $x \in W$, we can choose a suitable constant $c_{3}>0$ such that

$$
\left\langle\left(J-L_{0}\right) x, x\right\rangle \leq-c_{3}\|x\|^{2} .
$$

Then for any $x \in W \cap B_{s}$,

$$
I(x)=\frac{1}{2}\left\langle\left(J-L_{0}\right) x, x\right\rangle+I_{0}(x) \leq-\frac{1}{2} c_{3}\|x\|^{2}+c_{4}\|x\|=-\frac{1}{2} c_{3} r^{2}+c_{4} r .
$$

Choose $r$ such that $-(1 / 2) c_{3} r+c_{4}<0$ and take $\beta_{\infty}=\max \left\{\beta_{0},-(1 / 2) c_{1} r^{2}+c_{2} r\right\}$. Then the assumption (4) of Lemma 3.2 holds.

Therefore by Lemma 3.2, I possesses at least

$$
\#=\frac{1}{2}[\operatorname{dim}(V \cap W)-\operatorname{codim}(V+W)]
$$

geometrically different critical orbits in $I^{-1}\left(\left[\beta_{0}, \beta_{\infty}\right]\right)$.

From Lemma 2.4 and Definition 1.1, we can show that for $m$ large enough

$$
\begin{aligned}
\# & =\frac{1}{2}\left[\operatorname{dim} M^{-}\left(P_{m}\left(J-L_{0}\right) P_{m}\right)-\operatorname{dim} M^{-}\left(P_{m}\left(J-L_{\infty}\right) P_{m}\right)\right] \\
& =\frac{1}{2}\left[2 \mu\left(\alpha_{0}\right)-2 \mu\left(\alpha_{\infty}\right)\right] \\
& =\mu_{0}-\mu_{\infty} .
\end{aligned}
$$

Secondly, if $\mu_{\infty}-\mu_{0}-v_{0}>0$, then we replace $I$ by $-I$ and set

$$
V=M^{-}\left(J-L_{\infty}\right), \quad W=M^{+}\left(J-L_{0}\right) .
$$

With a similar argument to the case $\mu_{0}-\mu_{\infty}>0$, we can show that $-I$ satisfies the assumptions (1)-(5) of Lemma 3.2. Then $-I$ has at least $\#=(1 / 2)[\operatorname{dim}(V \cap W)-\operatorname{codim}(V+W)]$ geometrically different critical orbits in $I^{-1}\left(\left[\beta_{0}, \beta_{\infty}\right]\right)$. By Lemma 2.4, Definition 1.1, and for $m$ large enough,

$$
\begin{aligned}
\# & =\frac{1}{2}\left[\operatorname{dim} M^{-}\left(P_{m}\left(J-L_{\infty}\right) P_{m}\right)-\operatorname{dim}\left(M^{-}\left(P_{m}\left(J-L_{0}\right) P_{m}\right) \oplus M^{0}\left(P_{m}\left(J-L_{0}\right) P_{m}\right)\right)\right] \\
& =\frac{1}{2}\left[2 \mu\left(\alpha_{\infty}\right)-\left(2 \mu\left(\alpha_{0}\right)+2 v\left(\alpha_{0}\right)\right)\right] \\
& =\mu_{\infty}-\mu_{0}-v_{0} .
\end{aligned}
$$


Case (ii). Assume that $\mu_{0}-\mu_{\infty}>0$. We take the same subspaces $V$ and $W$ as in Case (i). By a similar discussion, we can prove that the assumptions of (1), (2), and (4) of Lemma 3.2 hold. By Lemma 3.3, I satisfies $(P S)_{c}$ condition on $E$.

By condition (ii) of Theorem 1.2, $I_{\infty}^{\prime}(x)$ is bounded, that is, $\exists c_{5}>0$ such that

$$
\left\|I_{\infty}^{\prime}(x)\right\| \leq c_{5}, \quad \forall x \in E .
$$

Moreover $I_{\infty}\left(x_{0}\right) \rightarrow-\infty$ as $x_{0} \in M^{0}\left(J-L_{\infty}\right)$ with $\left\|x_{0}\right\| \rightarrow+\infty$

Write $x=x_{+}+x_{0} \in V$, where $x_{+} \in M^{+}\left(J-L_{\infty}\right)$. We have

$$
\begin{aligned}
I(x) & =\frac{1}{2}\left\langle\left(J-L_{\infty}\right) x_{+}, x_{+}\right\rangle+I_{\infty}\left(x_{+}+x_{0}\right) \\
& >\frac{1}{2} c_{6}\left\|x_{+}\right\|^{2}+c_{5}\|x\|+I_{\infty}\left(x_{0}\right) .
\end{aligned}
$$

Thus the assumption (3) of Lemma 3.2 holds. Then by Lemma 3.2 and the proof of Case (i), I possesses at least $\mu_{0}-\mu_{\infty}$ geometrically different critical orbits in $I^{-1}\left(\left[\beta_{0}, \beta_{\infty}\right]\right)$. The subsequent proof is similar to that of Case (i). We omit the details. The proof is complete.

\section{References}

[1] T. Furumochi, "Existence of periodic solutions of one-dimensional differential-delay equations," The Tôhoku Mathematical Journal. Second Series, vol. 30, no. 1, pp. 13-35, 1978.

[2] W. J. Cunningham, "A nonlinear differential-difference equation of growth," The Proceedings of the National Academy of Sciences, vol. 40, no. 4, pp. 708-713, 1954.

[3] E. M. Wright, "A non-linear difference-differential equation," Journal für die Reine und Angezwandte Mathematik, vol. 194, no. 1, pp. 66-87, 1955.

[4] R. May, Stablity and Complexity in Model Ecosystems, vol. 9, Princeton University Press, Princeton, NJ, USA, 1973.

[5] S. N. Chow and H. O. Walther, "Characteristic multipliers and stability of symmetric periodic solutions of $\dot{x}=g(x(t-1))$," Transactions of the American Mathematical Society, vol. 307, no. 1, pp. 127-142, 1988.

[6] A. V. M. Herz, "Solutions of $\dot{x}=-g(x(t-1))$ approach the Kaplan-Yorke orbits for odd sigmoid $g$," Journal of Differential Equations, vol. 118, no. 1, pp. 36-53, 1995.

[7] J. L. Kaplan and J. A. Yorke, "Ordinary differential equations which yield periodic solutions of differential delay equations," Journal of Mathematical Analysis and Applications, vol. 48, no. 2, pp. $317-$ 324, 1974.

[8] J. L. Kaplan and J. A. Yorke, "On the stability of a periodic solution of a differential delay equation," SIAM Journal on Mathematical Analysis, vol. 6, no. 2, pp. 268-282, 1975.

[9] J. A. Yorke, "Asymptotic stability for one dimensional differential-delay equations," Journal of Differential Equations, vol. 7, no. 1, pp. 189-202, 1970.

[10] S. Chapin, "Periodic solutions of differential-delay equations with more than one delay," The Rocky Mountain Journal of Mathematics, vol. 17, no. 3, pp. 555-572, 1987.

[11] H. O. Walther, "Homoclinic solution and chaos in $\dot{x}(t)=f(x(t-1))$," Nonlinear Analysis: Theory, Methods \& Applications, vol. 5, no. 7, pp. 775-788, 1981.

[12] H. O. Walther, "Density of slowly oscillating solutions of $\dot{x}(t)=-f(x(t-1))$," Journal of Mathematical Analysis and Applications, vol. 79, no. 1, pp. 127-140, 1981.

[13] G. S. Jones, "The existence of periodic solutions of $f^{\prime}(x)=-\alpha f(x(t-1))\{1+f(x)\}$," Journal of Mathematical Analysis and Applications, vol. 5, no. 3, pp. 435-450, 1962.

[14] R. D. Nussbaum, "Periodic solutions of special differential equations: an example in nonlinear functional analysis," Proceedings of the Royal Society of Edinburgh. Section A, vol. 81, no. 1-2, pp. 131-151, 1978. 
[15] R. D. Nussbaum, "A Hopf global bifurcation theorem for retarded functional differential equations," Transactions of the American Mathematical Society, vol. 238, no. 1, pp. 139-164, 1978.

[16] R. D. Nussbaum, "Uniqueness and nonuniqueness for periodic solutions of $x^{\prime}(t)=-g(x(t-1))$," Journal of Differential Equations, vol. 34, no. 1, pp. 25-54, 1979.

[17] J. Li, X.-Z. He, and Z. Liu, "Hamiltonian symmetric groups and multiple periodic solutions of differential delay equations," Nonlinear Analysis: Theory, Methods E Applications, vol. 35, no. 4, pp. 457-474, 1999.

[18] J. Li and X.-Z. He, "Multiple periodic solutions of differential delay equations created by asymptotically linear Hamiltonian systems," Nonlinear Analysis: Theory, Methods \& Applications, vol. 31, no. 1-2, pp. 45-54, 1998.

[19] Z. Guo and J. Yu, "Multiplicity results for periodic solutions to delay differential equations via critical point theory," Journal of Differential Equations, vol. 218, no. 1, pp. 15-35, 2005.

[20] J. Llibre and A.-A. Tarța, "Periodic solutions of delay equations with three delays via bi-Hamiltonian systems," Nonlinear Analysis: Theory, Methods \& Applications, vol. 64, no. 11, pp. 2433-2441, 2006.

[21] S. Jekel and C. Johnston, "A Hamiltonian with periodic orbits having several delays," Journal of Differential Equations, vol. 222, no. 2, pp. 425-438, 2006.

[22] M. A. Han, "Bifurcations of periodic solutions of delay differential equations," Journal of Differential Equations, vol. 189, no. 2, pp. 396-411, 2003.

[23] P. Dormayer, "The stability of special symmetric solutions of $\dot{x}(t)=\alpha f(x(t-1))$ with small amplitudes," Nonlinear Analysis: Theory, Methods \& Applications, vol. 14, no. 8, pp. 701-715, 1990.

[24] F. E. Browder, "A further generalization of the Schauder fixed point theorem," Duke Mathematical Journal, vol. 32, pp. 575-578, 1965.

[25] S. N. Chow and J. Mallet-Paret, "The Fuller index and global Hopf bifurcation," Journal of Differential Equations, vol. 29, no. 1, pp. 66-85, 1978.

[26] J. Mawhin, "Periodic solutions of nonlinear functional differential equations," Journal of Differential Equations, vol. 10, no. 2, pp. 240-261, 1971.

[27] J. Mawhin, "Equivalence theorems for nonlinear operator equations and coincidence degree theory for some mappings in locally convex topological vector spaces," Journal of Differential Equations, vol. 12, no. 3, pp. 610-636, 1972.

[28] P. H. Rabinowitz, Minimax Methods in Critical Point Theory with Applications to Differential Equations, vol. 65 of CBMS Regional Conference Series in Mathematics, American Mathematical Society, Washington, DC, USA, 1986.

[29] J. Mawhin and M. Willem, Critical Point Theory and Hamiltonian Systems, vol. 74 of Applied Mathematical Sciences, Springer, New York, NY, USA, 1989.

[30] V. Benci, "On critical point theory for indefinite functionals in the presence of symmetries," Transactions of the American Mathematical Society, vol. 274, no. 2, pp. 533-572, 1982.

[31] L. O. Fannio, "Multiple periodic solutions of Hamiltonian systems with strong resonance at infinity," Discrete and Continuous Dynamical Systems, vol. 3, no. 2, pp. 251-264, 1997. 


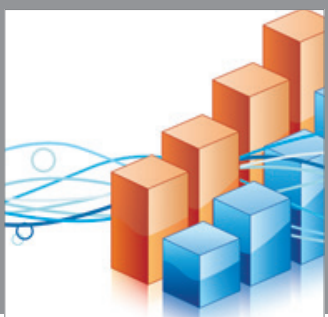

Advances in

Operations Research

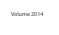

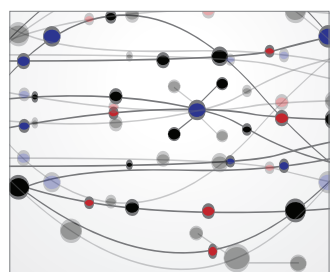

\section{The Scientific} World Journal
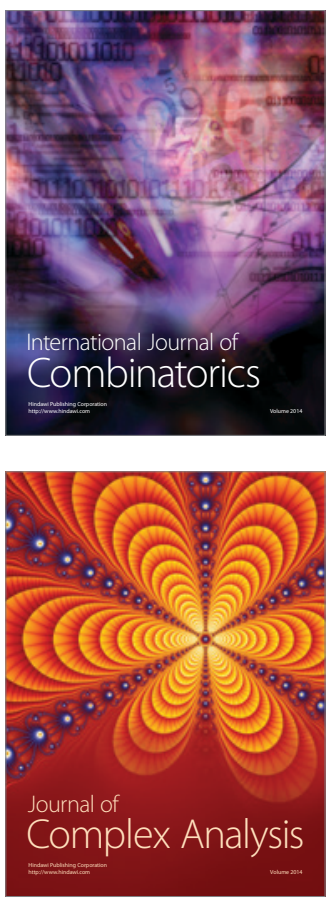

International Journal of

Mathematics and

Mathematical

Sciences
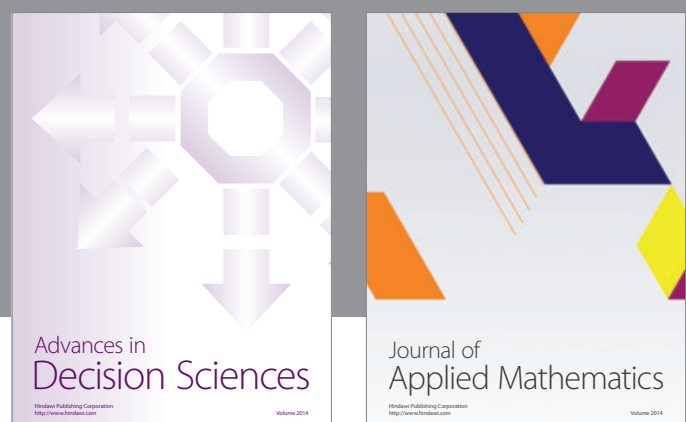

Journal of

Applied Mathematics
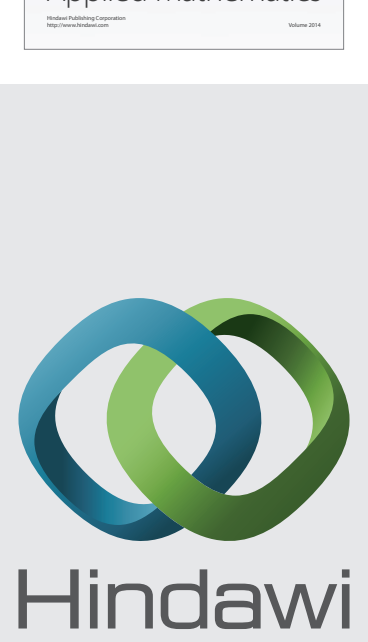

Submit your manuscripts at http://www.hindawi.com
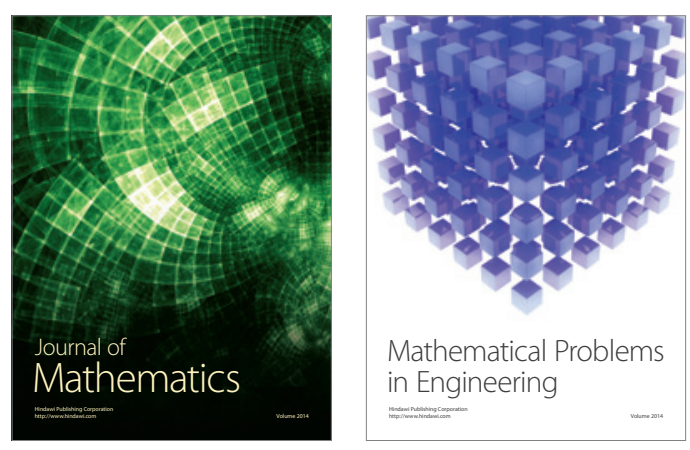

Mathematical Problems in Engineering
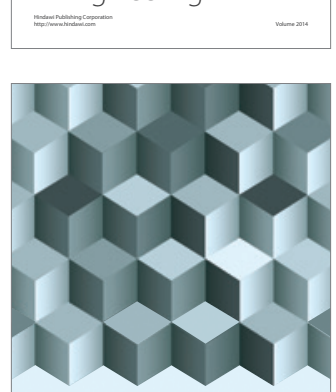

Journal of

Function Spaces
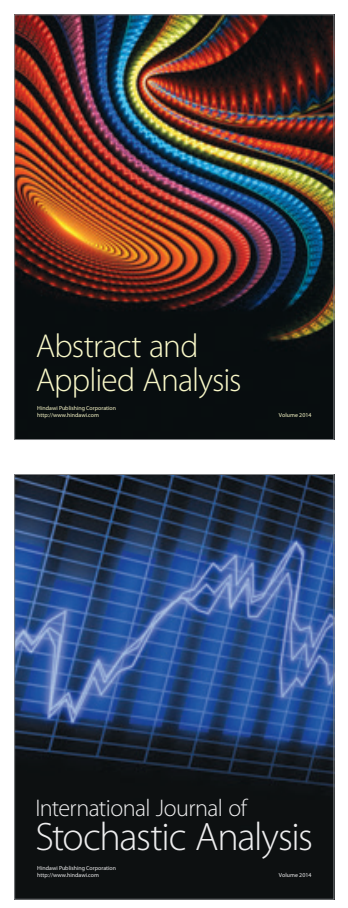

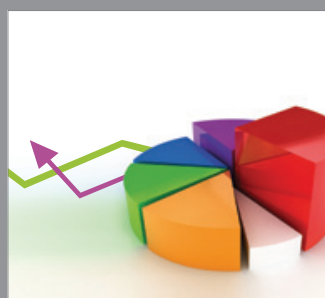

ournal of

Probability and Statistics

Promensencen
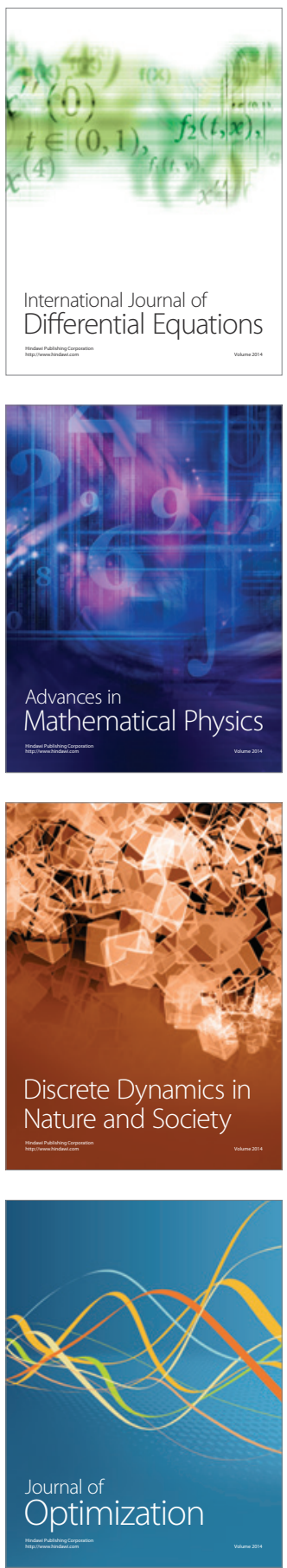